\title{
Addition of Rituximab to Chemotherapy Reduced the Rate of Surgery for Gastric-DLBCL Without Increasing Early Mortality
}

\author{
PHILIPPE PROUET ${ }^{1,2}$, SMITH GIRI $^{3}$, ERIC WIEDOWER $^{1,2}$, ANDREW FINTEL $^{4}$, \\ GEORGE YAGHMOUR $^{5}$, ELENA PAULUS LAMB ${ }^{6}$, JEREMIAH DENEVE ${ }^{7}$, MARTIN FLEMING ${ }^{7}$, \\ PAXTON DICKSON ${ }^{7}$, JASON C. CHANDLER $^{1,2}$ and MIKE G. MARTIN ${ }^{1,2}$ \\ ${ }^{1}$ The West Cancer Center, Germantown, TN, U.S.A.; \\ ${ }^{2}$ University of Tennessee Health Science Center Hematology/Oncology, Memphis, TN, U.S.A.; \\ ${ }^{3}$ Department of Medical Oncology, Yale School of Medicine, New Haven, CT, U.S.A.; \\ ${ }^{4}$ Blue Ridge Cancer Center, Salem, VA, U.S.A.; \\ ${ }^{5}$ Keck Hospital of USC, Los Angeles, CA, U.S.A.; \\ ${ }^{6}$ Fox Chase Cancer Center, Temple Health, Philadelphia, PA, U.S.A.; \\ ${ }^{7}$ Department of Surgery, University of Tennessee Health Science Center, Memphis, TN, U.S.A.
}

\begin{abstract}
Background: We evaluated surgical trends for gastric diffuse large B-cell lymphoma $(g D L B C L)$ before and after the approval of rituximab and whether an association of early mortality existed in patients treated after approval of rituximab. Patients and Methods: We utilized the Surveillance Epidemiology and End Results (SEER) 18 database to extract data on patients with gDLBCL diagnosed between 1983-2012. Primary site-specific cancer-directed surgery using SEER sitespecific surgical codes and annual trends were analyzed. Patients were analyzed before and after 2006, the year rituximab gained U.S. Food and Drug Administration approval. Results: Joinpoint trend analysis showed the sharpest decline in surgical rates between 2000-2010. Adjusted surgical rates computed using poisson regression declined from $54.4 \%$ in 1983 to $6.9 \%$ in 2012, with an annual percentage change of $-8.9 \%$ (95\% confidence interval $=-9.7 \%$ to $-8.3 \%$; $p$-value $<0.01)$. No significant mortality increase at 30 and 60 days was found. Conclusion: While rituximab appears to have significantly changed how surgery is utilized for patients with gDLBCL, early mortality was unchanged.
\end{abstract}

Over the past decades, the management of patients with gastric lymphoma has undergone significant changes,

Correspondence to: Philippe Prouet, MD, 7945 Wolf River Blvd, 3rd Floor Executive Suite, Germantown, TN 38138, U.S.A. E-mail: pprouet@westclinic.com

Key Words: Neoplasia, lymphoma, chemotherapeutic/ immunotherapeutic approaches, prognostication. shifting from surgical resection to treatment with chemotherapy with or without radiation. Historically, surgical intervention played a critical role in the multimodal management of gastric lymphoma. However, with the emergence of data demonstrating comparable outcomes with lower morbidity and better quality of life with chemotherapy and radiation, there has been a shift towards non-operative management for these patients (1-6). When comparing relapse-free survival (RFS) and overall survival (OS), Liu et al. showed no significant difference between patients treated with chemotherapy alone versus patients treated with surgery and adjuvant chemotherapy. Their results showed that the 5year RFS was $86 \%$ in the chemotherapy group and $78 \%$ in the surgery with adjuvant chemotherapy group $(p=0.94)$. The OS was $72.6 \%$ in the chemotherapy group and $77.8 \%$ in the surgery with adjuvant chemotherapy group $(p=0.40)(3)$. Willich et al. showed that 3-year and 5-year survival in patients treated with surgery was $88 \%$ versus $94 \%$ and $86 \%$ in patients treated conservatively $(p=0.350)$ indicating that an operative approach was not advantageous (4).

Rituximab, an engineered chimeric murine/human monoclonal antibody that targets CD20 antigens on Blymphocytes, gained Food and Drug Administration approval and patent for treatment of non-Hodgkin's lymphoma (NHL) in 1997 (7). A study by Coiffier et al. demonstrated that rituximab in combination with cyclophosphamide, doxorubicin, vincristine and prednisone (CHOP) chemotherapy was shown to improve OS (70\% vs. 57\%), event-free survival (57\% vs. $38 \%$ ) and complete response (52\%vs. 37\%) compared with CHOP alone in elderly patients with diffuse large B-cell lymphoma (8). In a Groupe d'Etude des Lymphomes de 
l'Adulte study from 2005, it was shown that complete response (47.5\% vs. $28 \%)$, event-free survival (47\% vs. 29\%), progression-free survival (54\% vs. 30\%), disease-free survival (66\% vs. $45 \%)$, and OS (58\% vs. $45 \%)$ were statistically significantly all in favor of rituximab-CHOP over CHOP alone (9). Another study by Habermann et al. published in 2006 showed that rituximab administered as induction or maintenance with $\mathrm{CHOP}$ chemotherapy significantly prolonged failure-free survival (53\% vs. 46\%) in older patients with DLBCL when compared to those who received CHOP alone (10). Rituximab with CHOP chemotherapy was also shown to be active and effective in treating gastric DLBCL (11).

An association with gastrointestinal perforation exists in patients with primary gastric NHL treated with rituximab $v s$. those treated with chemotherapy alone. The mean time to patients' reported symptoms was 6 days from the start of therapy (range $=1$ to 77 days) for documented gastrointestinal perforation. This risk is present in both follicular NHL and DLBCL (12). Rituximab has been shown to improve outcome in patients with NHL, although the risk exists for early mortality following treatment. As the role of surgery for gastric DLBCL has evolved from the primary management of the disease to the management of its complications, we sought to further define surgical trends and to explore whether the introduction of rituximab and the decreasing rate of primary surgery has influenced early mortality in patients with gastric DLBCL.

\section{Materials and Methods}

We utilized the Surveillance Epidemiology and End Results (SEER) 18 database (November 2014 submission) to extract relevant data on patients with gastric DLBCL diagnosed between 1983-2012. The SEER database currently collects and publishes cancer incidence and survival data from population-based cancer registries covering approximately $28 \%$ of the US population that includes $26 \%$ of African Americans, 38\% of Hispanics, $44 \%$ of American Indians and Alaska Natives, $50 \%$ of Asians, and $67 \%$ of Hawaiian/Pacific Islanders.

Eligible cases of gastric DLBCL were identified from the SEER 13 database using a combination of the International Classifications of Diseases for Oncology, 3rd edition (ICD-O-3) code for DLBCL (9680/3) and combining it with site code for stomach (C60.x) (13). We selected patients diagnosed in 1983 and later because of the lack of reliable surgical information on gastric cancer prior to 1983 and of Ann Arbor staging information prior to 1983.

We studied the receipt of primary site-specific cancer-directed surgery in this population using SEER site-specific surgery codes. Specific surgical types included partial, subtotal, or hemigastectomy, Billroth I or Billroth II, antrectomy, total or near total gastrectomy. Local tumor destruction using electrocautery, cryosurgery, laser, photodynamic therapy, polypectomy, incisional or excisional biopsy were not considered as primary site-specific cancer-directed surgery and were considered under the no-surgery group.

We studied the annual trends in the receipt of surgery among patients with gastric DLBCL. In February, 2006, the FDA approved rituximab in combination with $\mathrm{CHOP}$ or other anthracycline-based
Table I. Basic demographic characteristics of the study population before and after 2006, which is the year rituximab was approved by the Food and Drug Administration for first-line therapy of diffuse large $B$ cell lymphoma in combination with cyclophosphamide, doxorubicin, vincristine and prednisone.

\begin{tabular}{|c|c|c|c|}
\hline Variable & Before 2006 & After 2006 & $p$-Value \\
\hline Number of patients & 4124 & 1690 & \\
\hline Median age (range), years & $71(4-104)$ & $70(1-105)$ & 0.55 \\
\hline Age group, n $(\%)$ & & & 0.11 \\
\hline$<50$ years & $549(13.3 \%)$ & $214(12.7 \%)$ & \\
\hline $50-70$ years & $1436(34.8 \%)$ & $638(37.8 \%)$ & \\
\hline$>70$ years & $2139(51.9 \%)$ & $838(49.6 \%)$ & \\
\hline Gender, n (\%) & & $<0.01$ & \\
\hline Male & $1892(45.9 \%)$ & $698(41.3 \%)$ & \\
\hline Female & $2232(54.1 \%)$ & $992(58.7 \%)$ & \\
\hline Race, n (\%) & & & 0.01 \\
\hline White & $3350(81.2 \%)$ & $1344(79.5 \%)$ & \\
\hline Black & $302(7.3 \%)$ & $132(7.8 \%)$ & \\
\hline Other & $453(11.0 \%)$ & $194(11.5 \%)$ & \\
\hline Unknown & $19(0.5 \%)$ & $20(1.2 \%)$ & \\
\hline Ann Arbor stage, n (\%) & & & 0.03 \\
\hline I & $1840(44.6 \%)$ & $699(41.4 \%)$ & \\
\hline II & $814(19.7 \%)$ & $331(19.6 \%)$ & \\
\hline III & $233(5.6 \%)$ & $124(7.3 \%)$ & \\
\hline IV & $938(22.7 \%)$ & $417(24.7 \%)$ & \\
\hline Unknown & $299(7.3 \%)$ & $119(7.0 \%)$ & \\
\hline \multicolumn{4}{|l|}{ Radiation therapy, n (\%) } \\
\hline No & $3339(81 \%)$ & $1419(84 \%)$ & \\
\hline Yes & $699(16.9 \%)$ & $233(13.8 \%)$ & \\
\hline Unknown & $86(2.1 \%)$ & $38(2.2 \%)$ & 0.01 \\
\hline $\begin{array}{l}\text { Median year of } \\
\text { diagnosis (range) }\end{array}$ & $2000(1983-2006)$ & $2009(2007-2012)$ & $<0.01$ \\
\hline Surgery, n $(\%)$ & & & $<0.01$ \\
\hline No & $3109(75.4 \%)$ & $1565(92.6 \%)$ & \\
\hline Yes & $980(23.8 \%)$ & $108(6.4 \%)$ & \\
\hline Unknown & $35(0.8 \%)$ & $17(1.0 \%)$ & \\
\hline
\end{tabular}

chemotherapy as a first line therapy in patients with DLBCL. We compared the trends in surgical rates across this time period using two different methods. Firstly, unadjusted percentages (cases undergoing surgery per 100 patients) along with 95\% confidence intervals (CIs) (computed using bootstrapping methods) were computed for each year from the SEER database. Using Joinpoint regression program, (National Cancer Institute, Bethesda, MD, United States), the yearly trend in the surgical rate was evaluated. The program takes trend data and fits them to the simplest joinpoint model that the data allow. Changes in the trend of surgical rates were evaluated by Monte-Carlo permutation method. Similarly, the adjusted annual rate of surgery was computed using multivariate poisson regression method adjusting for age at diagnosis, gender, race and stage at diagnosis. Flexible but smooth rates were obtained with restricted cubic splines. We fitted models with one to five knots and compared the various models using Akake's information criterion, (14) which penalizes the more complicated models.

We also compared the impact of the introduction of rituximab on early mortality rates in our study population. Using the year 2006 as a cut-off, we studied the 30 - and 60-day mortality rates in the 


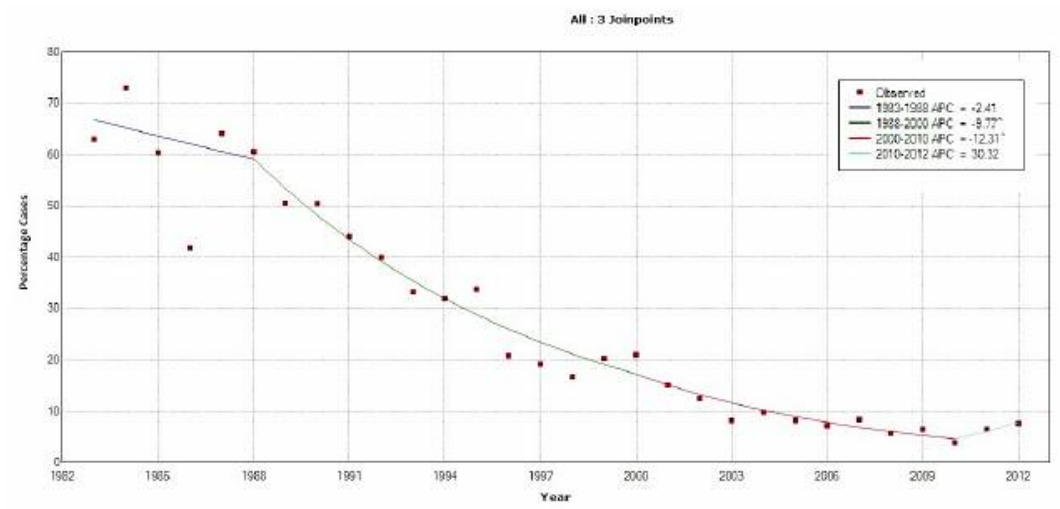

Figure 1. Trend in surgery for gastric diffuse large B-cell lymphoma from 1983-2012. Joinpoint trend analysis shows the decline in surgical rates for gastric diffuse large B-cell lymphoma, with the sharpest decline being between 2000 and $2010(p<0.01)$. Annual percentage change $=-12.31$, $95 \%$ confidence interval $=-16.8$ to $-7.6 ; p<0.01$.

study population. Multivariate logistic regression was used to study the impact of year of diagnosis (before and after 2006) on early mortality rates adjusting for age, sex, race and stage at diagnosis. The level of significance chosen was 0.05 . All $p$-values were twosided. No adjustments for multiple comparisons were made. Institutional Review Board waiver was obtained from the University of Tennessee Health Science Center prior to conducting this study.

\section{Results}

A total of 5,814 patients with gastric DLBCL were identified using the study criteria. This included 4,124 patients diagnosed prior to 2006 and 1,690 patients with gastric DLBCL diagnosed after 2006. The median age was 71 years (range 1 to 105 years), out of which $55.5 \%(n=3224)$ were males and $80.7 \%(n=4694)$ were Whites. The majority of patients had either stage I $(43.7 \% ; n=2539)$ or stage II $(19.7 \% ; n=1145)$ disease at the time of their diagnosis. A total of $16 \%$ of patients $(n=932)$ received some form of radiation therapy. As shown in Table I, patients diagnosed before and after 2006 were significantly different in terms of the distribution of gender, race, stage at diagnosis, use of radiation therapy and receipt of surgery.

A total of $1,088(18.7 \%)$ received primary cancer directed surgery during this time period. The proportion of patients with gastric DLBCL receiving surgery sharply declined from $63 \%$ in 1983 (34 out of 54 patients) to $7.6 \%$ (19 out of 250) in 2012. Joinpoint trend analysis revealed that the sharpest decline was seen between the years 2000-2010 [annual percentage change $(\mathrm{APC})=-12.31,95 \% \mathrm{CI}=-16.8$ to $-7.6 ; p<0.01]$ as illustrated in Figure 1. Similarly, the adjusted rate of surgery computed using poisson regression (adjusted for age, sex, race and stage at diagnosis) declined from $54.4 \%$ in 1983 to $6.9 \%$ in 2012 , with an APC of $-8.9 \%$ (95\% CI $=-9.7 \%$ to $-8.3 \%$; $p<0.01)$. Figure 2 shows the restricted cubic spline graph with annual trends in the surgical rates in the study population and shows the decline in primary site-specific surgery.

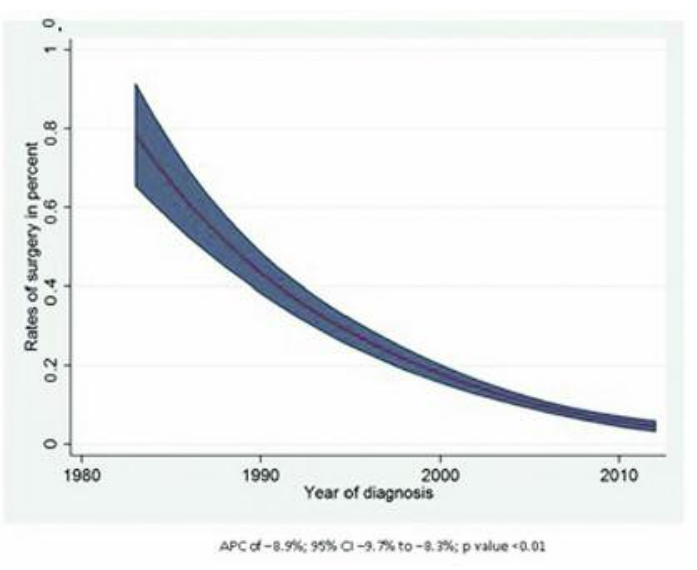

Figure 2. Adjusted rate of surgery computed using poison regression. The figure shows a decline in the surgical rate for gastric diffuse large B-cell lymphoma from 1983-2012. Annual percentage change $=-8.9$, $95 \%$ confidence interval $=-9.7$ to $-8.3 ; p<0.01$.

The 30-day mortality rate among patients diagnosed with gastric DLBCL prior to 2006 was $9.7 \%$ compared to $10.3 \%$ among patients diagnosed after $2006(p=0.48)$. Similarly, the 60 -day mortality rates were comparable in the two groups (16.3\% vs. $15.2 \%$; $p=0.29$ ). Multivariate logistic regression showed that the two groups (patients before and after 2006) were similar in terms of the 30-day mortality rate [odds $\operatorname{ratio}(\mathrm{OR})=0.97,95 \% \mathrm{CI}=0.79-1.18 ; p=0.79$ ] and the 60 -day mortality rate $(\mathrm{OR}=0.94,95 \% \mathrm{CI}=0.80-1.11 ; p=0.48)$ after adjusting for age, gender, race and stage at diagnosis.

\section{Discussion}

Outcomes for patients treated for gastric DLBCL have improved since rituximab was approved by the FDA to be used in combination with $\mathrm{CHOP}$ or other anthracyclines. A review 
Table II. Multivariate logistic regression showing the impact of year of diagnosis on 30 day mortality rates. There was no significant difference in the 30-day mortality in patients treated before 2006 compared to those treated after 2006.

\begin{tabular}{lccc}
\hline Variable & OR & $95 \%$ CI of OR & $p$-Value \\
\hline $\begin{array}{l}\text { Year of diagnosis } \\
\quad \text { Before 2006 (ref) }\end{array}$ & 1 & & \\
$\quad$ After 2006 & 0.97 & $0.79-1.18$ & 0.79 \\
Age group & & & \\
$\quad \quad$ 50 years (ref) & 1 & & \\
$\quad$ 50-70 years & 2.11 & $1.33-3.34$ & $<0.01$ \\
$\quad$ >70 years & 5.87 & $3.80-9.08$ & $<0.01$ \\
Gender & & & \\
$\quad$ Male (ref) & 1 & & \\
$\quad$ Female & 0.94 & $0.78-1.13$ & 0.52 \\
Race & & & \\
$\quad$ White (ref) & 1 & & \\
$\quad$ Black & 1.51 & $1.08-2.11$ & 0.02 \\
$\quad$ Other & 0.92 & $0.68-1.25$ & 0.59 \\
Ann Arbor stage & & & \\
$\quad$ I (ref) & 1 & & \\
II & 1.12 & $0.85-1.47$ & 0.41 \\
III & 1.25 & $0.84-1.84$ & 0.27 \\
IV & 2.14 & $1.72-2.66$ & $<0.01$ \\
\hline
\end{tabular}

OR: Odds ratio; CI: confidence interval; ref: reference.

of the literature also an showed improved survival. The literature also showed a decrease in the role of primary surgery for primary gastric NHL. It has been shown that surgery should be reserved for the treatment of patients who present with disease complications, such as bleeding or perforation, due to the innate morbidity of surgery (15-19). Our study was designed to explore whether the introduction of rituximab and the decreasing rates of primary surgery influenced early mortality in patients with gastric DLBCL. We found a decrease in the role of primary surgery for gastric DLBCL after the introduction of rituximab. Although surgical rates have declined, our study showed that there has been no statistically significant change in mortality since the introduction of rituximab.

There are several limitations to this study. This was a retrospective analysis from the SEER database. Time periods before and after the approval of rituximab in combination with CHOP for the treatment of gastric DLBCL were identified. Due to the nature of the SEER database, it is not known how many of the patients after 2006 were actually treated with rituximab, however, this was the standard of care. Pre-existing comorbidities and clinical lymphoma staging were not known along, nor their potential effects on early mortality in this study.

\section{Conclusion}

The period after the introduction of rituximab was associated with the sharpest decline in primary cancer-directed surgery
Table III. Multivariate logistic regression showing the impact of year of diagnosis on the 60-day mortality rate. There was no significant difference in the 60-day mortality in patients treated before 2006 compared to those treated after 2006.

\begin{tabular}{lccc}
\hline Variable & OR & $95 \% \mathrm{CI}$ & $p$-Value \\
\hline $\begin{array}{l}\text { Year of diagnosis } \\
\quad \text { Before 2006 (ref) }\end{array}$ & 1 & & \\
$\quad$ After 2006 & 0.94 & $0.80-1.11$ & 0.48 \\
Age group & & & \\
$\quad$ <50 years (ref) & 1 & & \\
50-70 years & 2.06 & $1.45-2.92$ & $<0.01$ \\
$\quad>70$ years & 5.95 & $4.26-8.31$ & $<0.01$ \\
Gender & & & \\
$\quad$ Male (ref) & 1 & & \\
$\quad$ Female & 1.03 & $0.89-1.19$ & 0.71 \\
Race & & & \\
$\quad$ White (ref) & 1 & & \\
$\quad$ Black & 1.62 & $1.23-2.12$ & $<0.01$ \\
$\quad$ Other & 0.90 & $0.70-1.15$ & 0.41 \\
Ann Arbor stage & & & \\
I (ref) & 1 & & 0.06 \\
II & 1.23 & $0.99-1.52$ & $<0.01$ \\
III & 1.63 & $1.19-2.24$ & $<0.01$ \\
IV & 2.67 & $2.23-3.19$ & \\
\hline
\end{tabular}

OR: Odds ratio; CI: confidence interval; ref: reference.

for gastric DLBCL. While rituximab is associated with perforation risk, no significant increase in 30- or 60-day mortality was seen in those treated after rituximab was approved for first-line therapy. While rituximab appears to have significantly changed how surgery is utilized in gastric DLBCL, early mortality is unchanged.

\section{Conflicts of Interest}

The Authors have no conflicts of interest to declare.

\section{References}

1 Feng L, Zhang G, Hu Z, Zou Y, Chen F, Zhang G and Tang L: Diagnosis and treatment of 81 patients with primary gastrointestinal lymphoma. J Cent South Univ Med Sci 34: 582588, 2009.

2 Lewin KJ, Ranchod M and Dorfman RF: Lymphomas of the gastrointestinal tract: a study of 117 cases presenting with gastrointestinal disease. Cancer 42: 693-707, 1978.

3 Liu HT, Hsu C, Chen CL, Chiang IP, Chen LT, Chen YC and Cheng AL: Chemotherapy alone versus surgery followed by chemotherapy for stage I/IIE large-cell lymphoma of the stomach. Am J Hematol 64: 175-179, 2000.

4 Willich NA, Reinartz G, Horst EJ, Delker G, Reers B, Hiddemann W, Tiemann M, Parwaresch R, Grothaus-Pinke B, Kocik J and Koch P: Operative and conservative management of primary gastric lymphoma: interim results of a German multicenter study. Int J Radiat Oncol Biol Phys 46: 895-901, 2000. 
5 Akhtar S and Maghfoor I: Rituximab plus CHOP for diffuse large-B-cell lymphoma. N Engl J Med 346: 1830-1831, 2002.

6 Fischbach W, Schramm S and Goebeler E: Outcome and quality of life favour a conservative treatment of patients with primary gastric lymphoma. Z Gastroenterol 49: 430-435, 2011.

7 Scott SD: Rituximab: a new therapeutic monoclonal antibody for non-Hodgkin's lymphoma. Cancer Pract 6: 195-197, 1998.

8 Coiffier B, Lepage E, Briere J, Herbrecht R, Herve T, Bouabdallah R, Morel P, Van Den Neste E, Salles G, Gaulard P, Reyes F, Lederlin P, Lederlin P and Gisselbrecht C: CHOP Chemotherapy plus rituximab compared with CHOP alone in elderly patients with diffuse large b-cell lymphoma. N Engl J Med 346: 235-242, 2002.

9 Paulus EM, Fleming MD, Hendrix AA, Deneve JL, Dickson PV, Mathew A, Martin MG and Munene G: The evolving role of surgery for gastric lymphoma: from curative resection to surgical management of complications. Am Surg 80: E322-324, 2014.

10 Popescu RA, Wotherspoon AC, Cunningham D, Norman A, Prendiville $\mathrm{J}$ and Hill ME: Surgery plus chemotherapy or chemotherapy alone for primary intermediate- and high-grade gastric non-Hodgkin's lymphoma: the Royal Marsden Hospital experience. Eur J Cancer 35: 928-934, 1999.

11 Wöhrer S, Püspök A, Drach J, Hejna M, Chott A and Raderer M: Rituximab, cyclophosphamide, doxorubicin, vincristine and prednisone (R-CHOP) for treatment of early-stage gastric diffuse large B-cell lymphoma. Ann Oncol 15: 1086-1090, 2004.

12 Rituximab [package insert]. San Fransico, CA: Genetech; 1997.

13 International Classifications of Diseases for Oncology, 3rd edition (ICD-O-3) code for DLBCL (9680/3) and combining it with site code for stomach (C60.x).
14 Akake's information criterion (AIC)

15 Cirocchi R, Farinella E, Trastulli S, Cavaliere D, Covarelli P, Listorti C, Desiderio J, Barberini F, Avenia N, Rulli A, Verdecchia GM, Noya G and Boselli C: Surgical treatment of primitive gastro-intestinal lymphomas: a systematic review. World J Surg Oncol 9: 145, 2011.

16 Aviles A, Nambo MJ, Neri N, Huerta-Guzman J, Cuadra I, Alvarado I, Castañeda C, Fernández R and González M: The role of surgery in primary gastric lymphoma: results of a controlled clinical trial. Ann Surg 240: 44-50, 2004.

17 Aviles A, Nambo MJ, Neri N, Talavera A and Cleto S: Mucosaassociated lymphoid tissue (MALT) lymphoma of the stomach: results of a controlled clinical trial. Med Oncol 22: 55-62, 2005.

18 Feugier P, Van Hoof A, Sebban C, Solal-Celigny P, Bouabdallah $\mathrm{R}$, Ferme C, Christian B and Lepage E: Long-term results of the R-CHOP study in the treatment of elderly patients with diffuse large B-Cell lymphoma: a study by the Groupe d'Etude des Lymphomes de 1'Adulte. J Clin Oncol 23: 4117-4126, 2005.

19 Habermann TM, Weller EA, Morrison VA, Gascoyne RD, Cassileth PA, Cohn JB, Dakhil SR, Woda B, Fisher RI, Peterson $\mathrm{BA}$ and Horning SJ: Rituximab-CHOP versus CHOP alone or with maintenance rituximab in older patients with diffuse large B-Cell lymphoma. J Clin Oncol 24: 3121-3127, 2006.

Received September 8, 2016

Revised December 17, 2016

Accepted January 9, 2017 